\title{
UN PLOMO CON DOBLE INSCRIPCION IBÉRICA LOCALIZADO EN BAETULO (BADALONA)
}

\author{
Montserrat Comas \\ Pepita Padrós \\ Javier Velaza
}

\section{Circunstancias del hallazgo}

La ciudad romana de Baetulo, situada en la costa catalana y debajo de la actual Badalona, es una fundación romana de inicios del siglo I a.C. Desde el año 1995 este yacimiento arqueológico está declarado Bien de Interés Cultural Nacional, y ello implica que las actuaciones urbanísticas que deban llevarse a cabo en la zona declarada han de contemplar la realización de la correspondiente intervención arqueológica previa y que sus resultados sean determinantes para cualquier tipo de actuación posterior.

En este sentido, a inicios de año 1999, se solicitó una licencia de obras para construir un bloque de viviendas con una planta sótano destinada a párking, en un solar situado en la calle Sant Josep de Rosés, esquina con las calles Sant Felipe y Mestre Nicolau. La calle Sant Josep de Rosés está situada en el casco antiguo de la ciudad, en el llamado barrio de Dalt la Vila, en el subsuelo del cual se encuentran los restos de la ciudad romana. El solar ocupaba $850 \mathrm{~m}^{2}$, de los cuales $630 \mathrm{~m}^{2}$ habían sido ya rebajados en los años 50 . El resto $-220 \mathrm{~m}^{2}$ - ocupado solo por casas en planta baja, tenía amplias expectativas arqueológicas, y por este motivo, se planteó su excavación en extensión. ${ }^{1}$

Los resultados confirmaron ampliamente dichas expectativas, ya que se pudieron documentar diversas fases de ocupación, de época romana, que abarcaban un período cronológico desde un momento anterior a mitad del siglo I a.C. hasta mediados del siglo II d.C.

En la primera fase de ocupación se hallaron diversas estructuras y elementos, entre los cuales destacan dos grandes silos de forma ovoide. Uno de ellos, que tenía unas dimensiones de $2,90 \mathrm{~m}$ de altura, $2 \mathrm{~m}$ de anchura máxima y una boca de 1,20 de diámetro, estaba colmatado por

${ }^{1}$ P.Padrós-M.Comas, Excavació arqueolòica al carrer Sant Josep de Rosés. Maig-juliol 1999. Ciutat romana de Baetulo (Badalona, Barcelonès). Memoria inédita depositada en el Servei d'Arqueologia de la Generalitat de Catalunya, Badalona 2001. 


\section{Comas - P. Padrós - J. Velaza}

un estrato de tierra que proporcionó una gran cantidad de materiales. Entre este material se localizó la pieza objeto de este estudio.

Se trata de una pieza de plomo en forma de disco de perfil muy irregular, que mide 5,9 cm de ancho por $6,9 \mathrm{~cm}$ de largo. Presenta una perforación circular en su extremo superior y dos cartelas rectangulares incisas en el centro de una de las caras, una encima de la otra, en cada una de las cuales aparece una inscripción con caracteres ibéricos. La cartela superior mide 3,3 cm de largo por 0,5 de ancho, y la inferior $3,2 \mathrm{~cm}$ de largo por 0,4 de ancho. En la parte inferior de la cara posterior, hay una placa de hierro de forma irregular, de $2,5 \mathrm{~cm}$ de ancho por $6,5 \mathrm{~cm}$ de largo, sin duda añadida a la pieza, de la que no podemos precisar su función, aunque posiblemente deba tratarse de una refacción.

La cronología del nivel que obliteraba el silo donde se halló esta pieza puede situarse a mediados del siglo I a.C. Esta datación se ha podido establecer gracias a la información proporcionada por los distintos materiales cerámicos hallados en este estrato de relleno, entre los cuales destaca la presencia de cerámica campaniense A de las formas Lamboglia 55 y Morel 113; cerámica campaniense B, de los tipos Lamboglia 3, 4 y 5/7; cerámica común de importación itálica; gran cantidad de cerámica gris de imitación campaniense, y ánforas Dressel 18 y Laietana 1. La cronología que presenta este material, unida a la total ausencia de terra sigillata itálica y de ánforas del tipo Pascual 1, cuya presencia se documenta en la ciudad de Baetulo hacia el 40 a.C., indica que la formación de este estrato debe situarse en un momento alrededor de la mitad del siglo I a.C.

Respecto a la identificación de esta pieza, no hemos encontrado ningún paralelo con el cual poder establecer una comparación. El hecho de que tenga una perforación en la parte superior indica que debió colgarse de algún lugar, ya sea porque así lo requiere su utilización específica, ya sea porque es una forma de guardarlo. Ello nos sugiere que quizás podría haberse utilizado a modo de etiqueta, colgado de algún gran recipiente, por ejemplo de un dolium, para identificar su contenido, su propietario, etc. Por otro lado, el peso considerable de este objeto - 265 gr - también podría indicar que se trata de un peso con inscripción de propietario, como algunos pesos de material pétreo con inscripción encontrados en Catalunya. Y aún existe una última posibilidad: por el tipo de cartela tan estrecha y pulida, así como por las letras inscritas con un trazo tan fino, podría identificarse como un sello que hubiese servido para marcar objetos cerámicos, o lacrar escritos o documentos.

De todas maneras, dejando al margen la utilización que hubiese tenido esta pieza, hay que constatar una circunstancia respecto a las dos inscripciones que presenta, y es que ambas cartelas contienen un mismo nombre situado en dirección inversa uno de otro, hecho que parece indicar que es importante que el nombre inscrito pueda ser leído, indistintamente, desde dos puntos. ${ }^{2}$

\footnotetext{
${ }^{2}$ Agradecemos a los doctores M. Mayer y J. Sanmartí sus opiniones sobre la posible función de esta pieza.
} 
Un plomo con doble inscripción ibérica localizado en Baetulo (Badalona)

\section{COMENTARIO EPIGRÁFICO}

Como ya se ha indicado más arriba, la pieza presenta en su cara anterior dos estampillados que, después de proceder a su estudio epigráfico y textual, resultan ser el mismo. El primero de ellos fue impreso en posición directa justo debajo del orificio de la pieza. El segundo, en posición inversa, unos centímetros más abajo. Las medidas de la primera estampilla son $3,3 \times 0,5 \mathrm{~cm}$ y las de la segunda $3,2 \times 0,4$ $\mathrm{cm}$. Como puede observarse, la diferencia entre ambas es mínima y puede justificarse por una pequeña inclinación en el momento de la aplicación del cuño. También el módulo de los signos, que ocupan completamente la altura de la estampilla, es idéntico $(0,5 / 0,4 \mathrm{~cm})$.

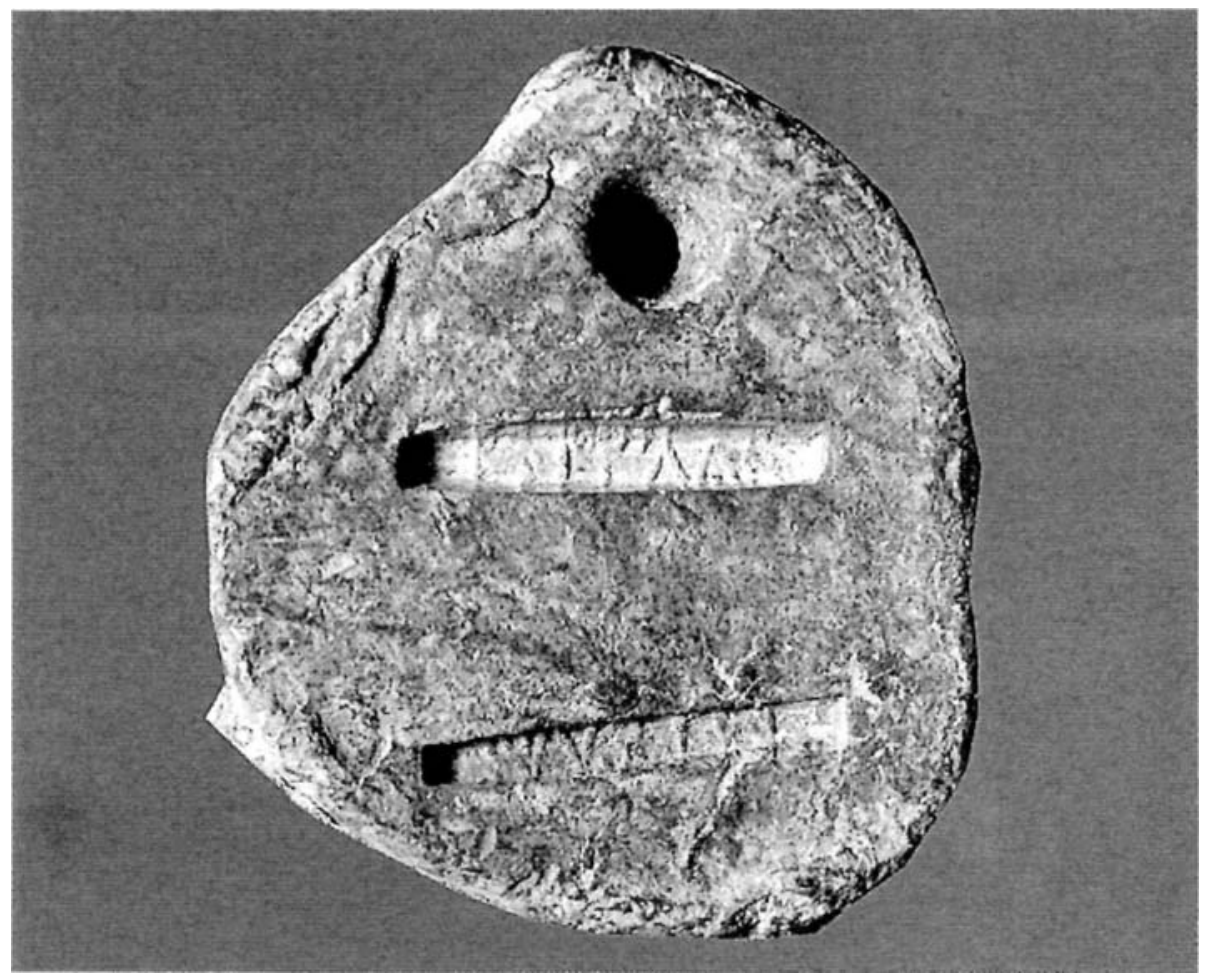

(Fotografía: A. Cartagena) 
M. Comas - P. Padrós - J. Velaza
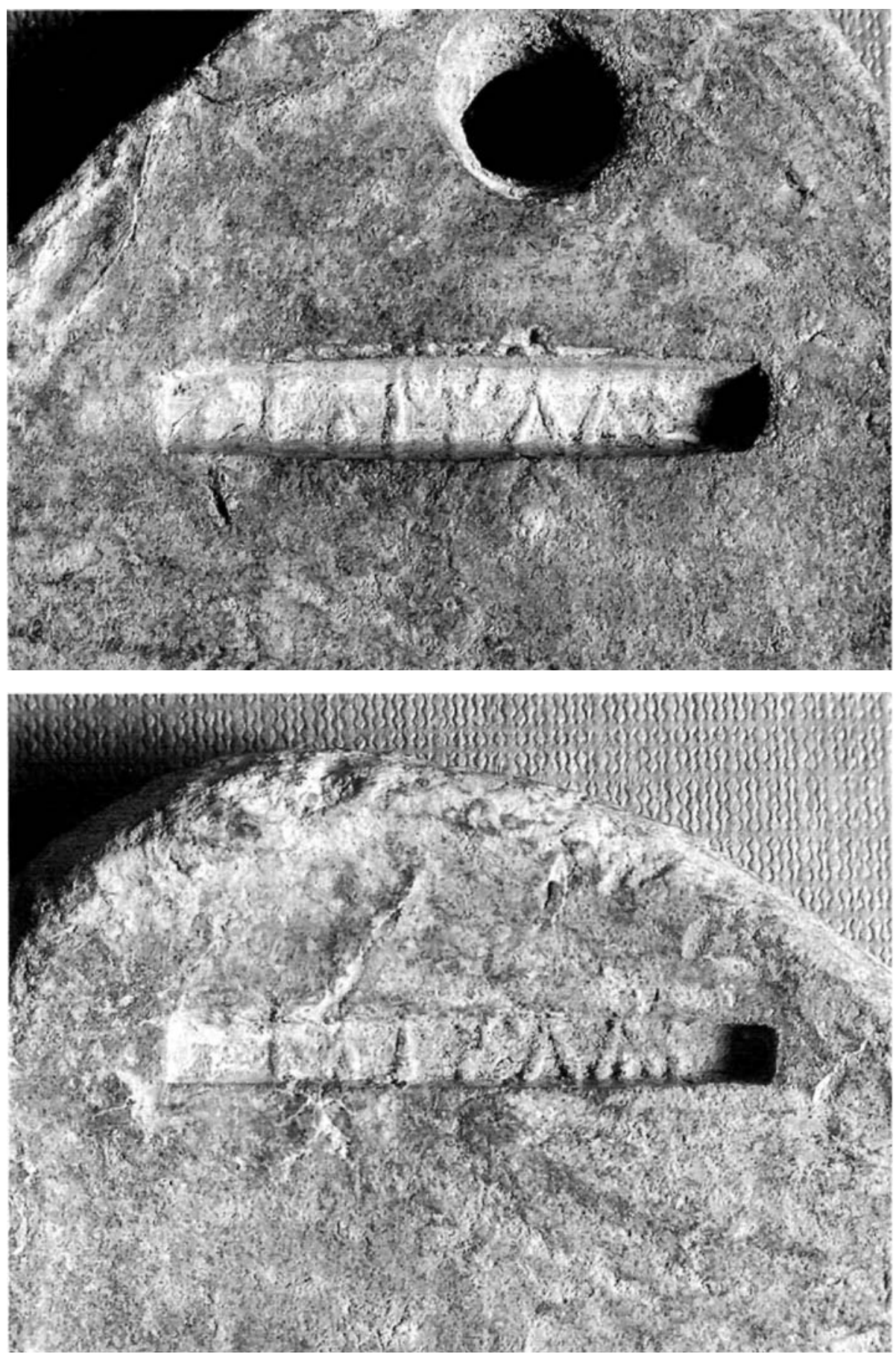

(Fotografías: A. Cartagena) 
Por otra parte, sin duda el hecho de que el cuño fuera el mismo es el causante de que ambos textos sean menos claros en su parte inicial, donde los signos aparecen más borrosos, en tanto que la inscripción gana en nitidez en su mitad final. De todos modos, una autopsia detenida permite proponer una lectura incuestionable del epígrafe como

\section{aiuniltun}

Desde el punto de vista paleográfico, las formas gráficas no ofrecen particularidades reseñables. Los signos obedecen al siguiente patrón en la clasificación de Untermann: ${ }^{3}$ a1, i1, u1, n1, 11, tu2.

Por lo que se refiere al texto, la forma aiuniltun no plantea mayores problemas para ser entendida como un nombre personal. Por otro lado, sus formantes aiun- e -iltun son suficientemente conocidos como formadores de antropónimos: para el primero, que sólo parece funcionar como primer elemento, pueden recordarse aiun (G.14.1), aiun-eskeŕ (E.1.308), aiun-i (F.11.1), aiun-in (E.12.1), aiun-ortin-ika (C.21.6, A), aiun-ortin-iku (C.21.6, B-2). Para el segundo, que funciona indiferentemente como primero o segundo, pueden compararse aloŕiltun (D.10.1, 1), anboś-iltun-u (F.20.1, A-I, 3), bekon-iltun (F.21.1, A-1), iltun-eśker (E.6.3), iske-iltun (F.21.1, A-9), labeis-iltun-iŕ (F.20.1, A-I, 1), neŕe-iltun (F.11.6, 2), selkim-iltun (F.21.1, A-4), uniltun (C.0.2).

Este análisis invita a suponer que el nombre personal corresponde al del productor o dueño de la factoría o taller que había producido o elaborado aquello que se enviaba marcado con la etiqueta.

Monserrat Comas

Museu de Badalona

e-mail:mcomas@museubdn.es
Pepita Padrós

Museu de Badalona e-mail:ppadros@museubdn.es

Javier Velaza

Universitat de Barcelona

e-mail:velaza@lingua.fil.ub.es

${ }^{3}$ J.Untermann, Monumenta Linguarum Hispanicarum III.1, Wiesbaden 1990, pp. 246-247. 